\title{
Study of the $B$-site ion behaviour in the multiferroic perovskite bismuth iron chromium oxide
}

Cite as: J. Appl. Phys. 123, 154104 (2018); https://doi.org/10.1063/1.5020305

Submitted: 21 December 2017 . Accepted: 31 March 2018 . Published Online: 17 April 2018

Bethany R. McBride, Jonathon Lieschke, Adam Berlie, David L. Cortie, Helen Y. Playford, Teng Lu, Narendirakumar Narayanan, Ray L. Withers, Dehong Yu, and Yun Liu
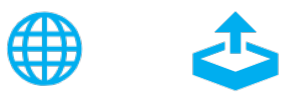

\section{ARTICLES YOU MAY BE INTERESTED IN}

Structural investigation of (111) oriented $\left(\mathrm{BiFeO}_{3}\right)_{(1-\mathrm{x}) \Lambda} /\left(\mathrm{LaFeO}_{3}\right)_{\mathrm{x}_{\Lambda}}$ superlattices by $\mathrm{X}$-ray diffraction and Raman spectroscopy

Journal of Applied Physics 123, 154103 (2018); https://doi.org/10.1063/1.5032085

Effect of optical damage resistant dopants on the dielectric properties of LiNbO3: Insight from broadband impedance spectroscopy and Raman scattering

Journal of Applied Physics 123, 154105 (2018); https://doi.org/10.1063/1.5021758

Giant electrocaloric effect in a cracked ferroelectrics

Journal of Applied Physics 123, 154102 (2018); https://doi.org/10.1063/1.5004203

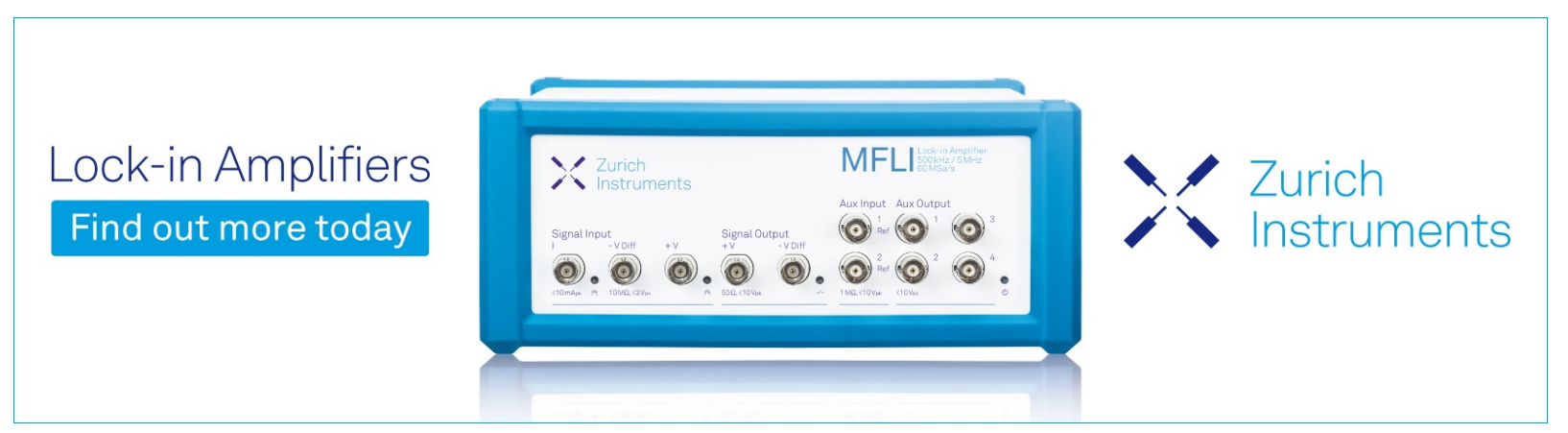




\title{
Study of the $B$-site ion behaviour in the multiferroic perovskite bismuth iron chromium oxide
}

\author{
Bethany R. McBride, ${ }^{1}$ Jonathon Lieschke, ${ }^{1}$ Adam Berlie,,${ }^{1,2,3,4, a)}$ David L. Cortie,,${ }^{1,2}$ \\ Helen Y. Playford, ${ }^{3}$ Teng Lu, ${ }^{1}$ Narendirakumar Narayanan, ${ }^{1,2}$ Ray L. Withers, ${ }^{1}$ Dehong Yu, ${ }^{2}$ \\ and Yun Liu ${ }^{1, a)}$ \\ ${ }^{1}$ Research School of Chemistry, The Australian National University, Canberra, ACT 2601, Australia \\ ${ }^{2}$ Australian Centre for Neutron Scattering, The Australian Nuclear Science and Technology Organisation, \\ Lucas Heights, NSW 2234, Australia \\ ${ }^{3}$ ISIS Neutron and Muon Facility, STFC Rutherford Appleton Laboratory, Harwell Science and Innovation \\ Campus, Oxfordshire OX11 OQX, United Kingdom \\ ${ }^{4}$ RIKEN Nishina Centre for Accelerator-Based Science, 2-1 Hirosawa, Wako, Saitama 351-0198, Japan
}

(Received 21 December 2017; accepted 31 March 2018; published online 17 April 2018)

\begin{abstract}
A simple, near-ambient pressure solid-state method was developed to nominally synthesize $\mathrm{BiFe}_{0.5} \mathrm{Cr}_{0.5} \mathrm{O}_{3}$. The procedure allowed the gram-scale production of multiferroic samples with appreciable purity and large amounts of $\mathrm{Cr}$ incorporation that were suitable for systematic structural investigation by neutron, X-ray, and electron diffraction in tandem with physical characterization of magnetic and ferroelectric properties. The rhombohedrally distorted perovskite phase was assigned to the space group $R 3 \mathrm{c}$ by way of $\mathrm{X}$-ray and neutron powder diffraction analysis. Through a combination of magnetometry and muon spin relaxation, it is evident that there is magnetic ordering in the BFCO phase consistent with G-type antiferromagnetism and a $\mathrm{T}_{\mathrm{N}} \sim 400 \mathrm{~K}$. There is no clear evidence for chemical ordering of $\mathrm{Fe}$ and $\mathrm{Cr}$ in the $B$-site of the perovskite structure and this result is rationalized by density functional theory and bond valence simulations that show a lowered energy associated with a $B$-site disordered structure. We believe that our contribution of a new, low-complexity method for the synthesis of BFO type samples, and dialogue about realising certain types of ordering in oxide perovskite systems, will assist in the further development of multiferroics for next-generation devices. Published by AIP Publishing.
\end{abstract}

https://doi.org/10.1063/1.5020305

\section{INTRODUCTION}

Materials that simultaneously exhibit both ferromagnetism and ferroelectricity attract a great deal of attention for their applications in next-generation multifunctional devices. Manipulation of the ordered states and the resulting properties of these "multiferroic" materials can theoretically be used to execute valuable functions like magnetoelectric memory storage and solid-state logic. ${ }^{1}$

For a long time, bismuth ferrite $\left(\mathrm{BiFeO}_{3}\right)$ has been the flagship candidate for such advanced applications. Through extensive investigation, $\mathrm{BiFeO}_{3}$ has been verified as a multiferroic material, exhibiting both ferroelectricity and G-type, canted-antiferromagnetism at room temperature. ${ }^{2}$ However, researchers have also noted a number of hindrances to its practical usage, such as low net magnetization, high leakage currents, ${ }^{2}$ processing dependent properties ${ }^{3}$ and appreciable difficulties with purity control in synthesis. ${ }^{4-6}$ The origin of the multiferroicity in $\mathrm{BiFeO}_{3}$ is attributed to the pairing of a $\mathrm{d}^{0}$ cation in the $A$-site of its $R 3 c, A B \mathrm{O}_{3}$ perovskite structure (e.g., $\mathrm{Bi}^{3+}$ ) with a $\mathrm{d}^{\mathrm{n}}$ cation in the $B$-site (e.g., $\mathrm{Fe}^{3+}$ ). The former is spontaneously polarisable, while the latter can be involved in magnetic ordering. ${ }^{7}$ In this spirit, researchers have turned to identifying other perovskites that meet these

\footnotetext{
${ }^{\text {a) }}$ Authors to whom correspondence should be addressed: adam.berlie@stfc.ac.uk and yun.liu@anu.edu.au
}

prerequisites in the hope that they show better practical properties than $\mathrm{BiFeO}_{3}$.

To date, a variety of computational and experimental studies have predicted other viable multiferroic perovskites. One such promising candidate, bismuth iron chromium oxide $\left(\mathrm{Bi}_{2} \mathrm{FeCrO}_{6}, \mathrm{BFCO}\right)$, was initially suggested by ab initio studies. ${ }^{7,8}$ The predicted material had a "double-perovskite," $B$-site ordered structure with a space group symmetry of $R 3$ (O-phase) in which the occupation of the $B$-site alternated between $\mathrm{Fe}$ and $\mathrm{Cr}$ along the parent $[111]_{\mathrm{p}}$ (subscript $\mathrm{p}$ for parent) pseudo-cubic direction, leading to ferrimagnetism (net magnetization of $160 \mathrm{emu} / \mathrm{cm}^{3}, \sim 18 \mathrm{Am}^{2} / \mathrm{kg}$ ) and ferroelectricity (polarization $79.6 \mu \mathrm{C} / \mathrm{cm}^{2}$ ) in its ground state and thus could potentially be more practically useful than $\mathrm{BiFeO}_{3}$. Understandably, significant effort has since been expended in experimentally realising this impressive result in the hope that this magnetic and polar ordering persists at room temperature.

By a range of experimental methods, syntheses of thin films, powder and bulk samples of BFCO have been conducted with varying degrees of success in sample purity and observed properties. The reported room temperature properties span orders of magnitude, with measured saturation magnetization from $\sim 5-150 \mathrm{emu} / \mathrm{cm}^{3}$ and remanent polarisation from $\sim 0.445-53 \mu \mathrm{C} / \mathrm{cm}^{2}$ observed, ${ }^{9-12}$ all falling short of the initial ab initio predictions. From the theoretical study, the key to the finding of high magnetization in 
particular is the degree of $B$-site chemical ordering, i.e., having a double-perovskite structure. However, across a broad range of synthesis techniques, fully disordered structures (space group R3c, D-phase), ${ }^{12}$ mostly ordered structures, ${ }^{13}$ and mixed other orderings ${ }^{10}$ have been reported with full ordering seemingly only attainable by artificial layering. ${ }^{14}$ These variable synthesis results are reflected in the large range in observed properties. Physically achieving the requisite fully $B$-site ordered structure is evidently quite difficult, but experimentally verifying this order is also problematic.

A $\mathrm{Fe} / \mathrm{Cr}$, randomly disordered structure formally has $R 3 c$ space group symmetry, whereas a fully (or partially) Fe/ $C r$ ordered structure formally has only $R 3$ space group symmetry. In diffraction experiments, the $c$-glide symmetry operations of the former formally give rise to the systematic extinction conditions $\mathrm{F}\left(\langle h h l\rangle_{\mathrm{R}}{ }^{*}\right) \equiv[h h l]_{\mathrm{R}} *, \quad[l h h]_{\mathrm{R}}{ }^{*}$ and $\left([h l h]_{\mathrm{R}} *\right.$ ) all $=0$ unless $l$ is even. (The subscript $\mathrm{R}$ here refers to the primitive, i.e., rhombohedral, setting of $R 3 c$, while the * symbol denotes a reciprocal space reflection.) Note that if $h=0$ above then $\mathrm{F}\left(\mathbf{c}_{\mathrm{R}}{ }^{*} \equiv l / 2[11-1]_{\mathrm{p}}{ }^{*}, l \mathbf{a}_{\mathrm{R}}{ }^{*} \equiv l / 2[-111]_{\mathrm{p}}{ }^{*}\right.$, and $\left.l \mathbf{b}_{\mathrm{R}} * \equiv l / 2[1-11]_{\mathrm{p}}{ }^{*}\right)$ also $=0$ unless $l$ is even. Likewise, note that if $l=h$ above then $\mathrm{F}\left([l l l]_{\mathrm{R}} * \equiv l / 2[111]_{\mathrm{p}} *\right)$ also $=0$ unless $l$ is even. Thus, all four $\langle 1 / 2,1 / 2,1 / 2\rangle_{\mathrm{P}}{ }^{*}$ pseudo-cubic, parent reflections are forbidden by $R 3 c$ space group symmetry. This explains why, to date, the most common method of assessing $\mathrm{Fe} / \mathrm{Cr}$ ordering has been the detection of the $c$-glide breaking $\langle 1 / 2,1 / 2,1 / 2\rangle_{\mathrm{P}} *$ pseudo-cubic parent reflection in $\mathrm{X}$-ray diffraction studies on thin films. The relative intensity of this peak to the parent $\langle 1,1,1\rangle_{\mathrm{P}}{ }^{*}$ reflection from sample to sample has likewise been attributed to the variability of the "degree of $\mathrm{Fe} / \mathrm{Cr}$ ordering." 13

Electron diffraction has its own limitations, chiefly the difficulty in isolating the appropriate zone axis orientation from a "single crystal" region of a polycrystalline sample (to be outlined further in this report). Neutron powder diffraction, on the other hand, can provide excellent elemental contrast between $\mathrm{Fe}$ and $\mathrm{Cr}$ (the coherent neutron scattering lengths for $\mathrm{Fe}=9.45 \mathrm{fm}$ and for $\mathrm{Cr}=3.64 \mathrm{fm}$ ) as well as the possibility of directly determining the magnetic structure. Both are needed to pinpoint the $B$-site chemical and magnetic ordering behaviour. The main reason for not employing neutron diffraction so far seems to be the apparent difficulty in making sufficiently high purity, large quantity samples of BFCO. To date, only a small number of powder samples with a pure phase have been obtained. These required high pressure synthesis methods ${ }^{10,12}$ which do not allow for sample production in large quantities. Reported attempts at solid state syntheses of BFCO, which in theory can produce bulk samples, seem to result in considerable amounts of undesired impurity phases when performed at ambient pressure..$^{15,16}$

In this work, we successfully use a near-ambient pressure environment to synthesize bulk BFCO ceramic samples using a solid state reaction. The resultant BFCO samples made with appreciable purity at the gram-scale have a rhombohedral perovskite average structure. A systematic investigation by neutron, X-ray, and electron diffraction in tandem with magnetic and ferroelectric property determination is conducted to elucidate the $B$-site behaviour. We couple these results with computational methods to suggest why the double-perovskite structure and associated high magnetization are so elusive in both synthesis and determination of BFCO. We hope our commentary on the link between the structure and observed magnetic properties in multiferroic BFCO will assist in the further development of multiferroics for next-generation devices.

\section{METHODS}

\section{A. Sample preparation}

Dried powders of $\mathrm{Bi}_{2} \mathrm{O}_{3}$ (Alfa Aesar 99\%), $\mathrm{Fe}_{2} \mathrm{O}_{3}$ (Sigma-Aldrich, $>99 \%$ ), and $\mathrm{Cr}_{2} \mathrm{O}_{3}$ (Koch-light Laboratories, 99.999\%) were mixed in ethanol using a planetary ball mill. Teflon canisters containing the reactants, solvent, and yttria stabilized zirconia balls $(2-12 \mathrm{~mm})$ were rotated at $\sim 20 \mathrm{~Hz}$ for $10-24 \mathrm{~h}$. The resulting sub-micron powders were dried at $100^{\circ} \mathrm{C}$, ground using a mortar and pestle, and then cold pressed into pellets $(\sim 0.7 \mathrm{~g}$ each $)$ at $\sim 63 \mathrm{MPa}$ of pressure in a $12 \mathrm{~mm}$ stainless steel die. Quick fit glassware was fashioned into a loose sealing quartz glass crucible [Fig. 1(a)] to facilitate a near-but-above atmospheric pressure environment for the material fabrication. The samples, inside the crucibles, were heat-treated in a muffle furnace to a temperature of $860^{\circ} \mathrm{C}$, and then held for 30-60 min before quenching the vessel to air.

\section{B. Structure investigation}

X-ray powder diffraction (XRPD) patterns were collected on a PANalytical Empyrean diffractometer utilizing a $\mathrm{Cu}$ target. Transmission Electron Diffraction (JEOL 2100F FEGTM) patterns were collected on a finely ground sample loaded onto a carbon-coated copper grid in an attempt to observe evidence for $\mathrm{Fe}-\mathrm{Cr}$ ordering. Neutron diffraction data were initially collected on the high resolution powder diffractometer "Echidna" (Australian Nuclear Science and Technology Organization, wavelength $1.622 \AA$ ) and later measured on the Polaris time-of-flight neutron diffractometer at ISIS (the UK's spallation neutron and muon source). The powdered sample was loaded into a silica glass capillary of $1.5 \mathrm{~mm}$ inner diameter, and placed inside the Polaris
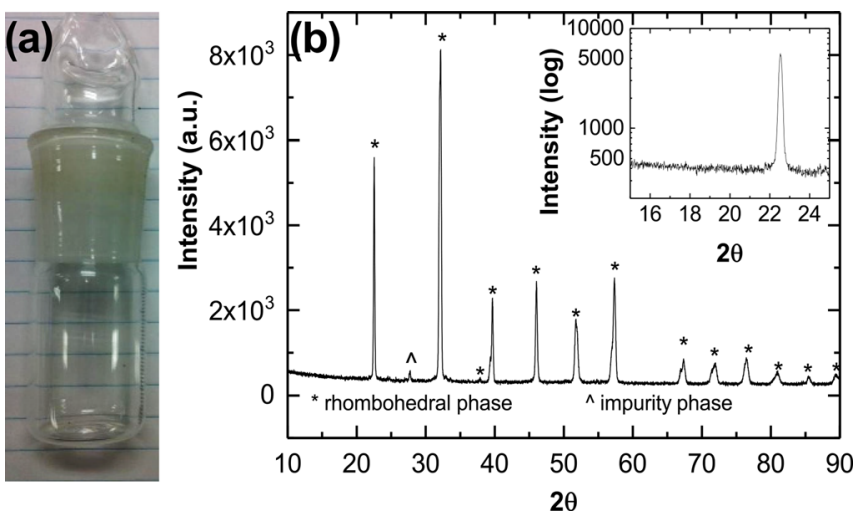

FIG. 1. (a) Quartz crucible fashioned from quick-fit glassware. (b) XRPD pattern of the BFCO sample with a rhombohedral phase indicated. Inset: low angle region of the diffraction pattern with intensity plotted on a $\log$ scale, showing the clear absence of the $\langle 1 / 2,1 / 2,1 / 2\rangle_{\mathrm{P}} *$ peak (expected $\sim 19.4^{\circ}$ ). 
instrument. A "low-background" double-skinned resistive element furnace with vanadium-foil element and heatshields and no outer vacuum jacket was used to heat the sample up to a maximum temperature of $600 \mathrm{~K}$. Diffraction patterns suitable for Rietveld refinement ( $\sim 4 \mathrm{~h}$ of counting time) were collected at room temperature and $500 \mathrm{~K}$ under zero magnetic field. The raw data were focused and normalised using the program Mantid ${ }^{17}$ and Rietveld refinement was carried out using the program GSAS. ${ }^{18,19}$

\section{Physical property characterization}

Magnetic susceptibility data were collected using a Quantum Design magnetic property measurement system (MPMS) with a $7 \mathrm{~T}$ superconducting solenoid magnet. Zerofield cooled (ZFC) and field cooled (FC) data were collected in a $1 \mathrm{~T}$ field. Magnetization as a function of field measurements was also collected up to $7 \mathrm{~T}$. An oven option was used to obtain magnetization data up to $600 \mathrm{~K}$ in a field of $0.0175 \mathrm{~T}$. A muon spin relaxation $(\mu \mathrm{SR})$ experiment was conducted using the ARGUS spectrometer of the RIKENRAL Muon Facility, under zero field. Due to the small size of the sample, the measurement was conducted in fly-past mode where a $1 \mathrm{~cm}^{2}$ silver foil packet containing the sample was mounted on a helium flow cold-finger cryostat. The data were collected using a double pulse mode, where each pulse is separated by $324 \mathrm{~ns}$ and has a FWHM of $70 \mathrm{~ns}$.

Electrical polarization on the local scale was measured using piezoresponse force microscopy (PFM, Cypher, Asylum Research). Pristine domains were imaged in Dual AC Resonance Tracking mode using an Olympus AC240TM probe as the cantilever with a contact force of $\sim 100 \mathrm{nN}$ and a driving voltage of $0.3 \mathrm{~V}$. Switching PFM spectra were collected using an applied DC bias of $\pm 50 \mathrm{~V}$.

\section{Simulation}

The final structures were validated in terms of their global instability index (GII) using the SOFTBV bond valence sum method. ${ }^{20,21}$ Density Functional Theory (DFT) calculations were performed using the full-potential linearized augmented plane wave plus local orbital method as implemented in the WIEN2k code ${ }^{22}$ using the PBE-GGA exchange-correlation energy functional. ${ }^{23}$ To include the effects due to the strong onsite electron-electron Coulomb repulsion in the $\mathrm{Fe}$ and $\mathrm{Cr}$ d-bands, the $\mathrm{GGA}+\mathrm{U}$ method was also applied, using the Self-interaction Correction (SIC) method, ${ }^{24}$ with the values of $\mathrm{U}$ and $\mathrm{J}$ adopted from Ref. 8 . The total energy was calculated for the antiferromagnetic alignment of planes of $\mathrm{Fe}$ and $\mathrm{Cr}$ spins along the [111] pseudo-cubic direction, in either the ordered or partially disordered chemical cell based on the $R 3$ space group, translated into the $P 1$ space group for the purposes of the magnetic structure. The supercell thus contained $6 B$-sites, and 30 atoms overall. The non-overlapping radial muffin tin sizes $\left(\mathrm{R}_{\mathrm{MT}}\right)$ for the individual species were: $\mathrm{Bi}$ (2.3 a.u.), Fe (1.94 a.u.), $\mathrm{Cr}$ (1.94 a.u.), and $\mathrm{O}$ (1.67 a.u.). The calculations were converged using an $\mathrm{R}_{\mathrm{MT}} \mathrm{K}_{\mathrm{Max}}$ value of 8 , where $\mathrm{K}_{\mathrm{Max}}$ denotes the largest plane wave vector, and a k-point mesh containing 64 points was used. The starting structure was adopted from the theoretical double-perovskite, using the experimental lattice constants, and ionic relaxation of internal coordinates was performed to converge forces to $<1$ $\mathrm{mRy} /$ Bohr. A disordered variant of the structure was considered by interchanging one of the $\mathrm{Fe}$ and $\mathrm{Cr}$ sites, and comparing the properties with and without ionic relaxation.

\section{RESULTS AND DISCUSSION}

\section{A. Near-ambient pressure synthesis}

In order to perform a comprehensive characterization of the BFCO system, it is necessary to produce samples on the gram-scale. The most straight forward way to do so is to use solid-state synthetic methods. However, BFO based materials have historically presented many challenges for the solidstate synthesis approach, due to issues like the volatility of $\mathrm{Bi}_{2} \mathrm{O}_{3},{ }^{25}$ differences in diffusion rates of reactants, ${ }^{6}$ reactant purity driven deviations from a binary phase system, ${ }^{5}$ and the thermodynamic ease of formation of parasitic phases. ${ }^{4,25}$ The influence of adding more elements $(\mathrm{Cr})$ on the already complex $\mathrm{Bi}_{2} \mathrm{O}_{3}$ and $\mathrm{Fe}_{2} \mathrm{O}_{3}$ system as noted in these references unsurprisingly makes the synthesis of BFCO very challenging. In fact, the small amount of powder BFCO samples reported to date were obtained using pressures of 4-6 GPa during synthesis. ${ }^{10,12}$ To the best of our knowledge, this paper is the first to propose this near-ambient pressure solidstate method to produce high-purity, relatively large-scale samples of bulk BFCO.

The success of our method hinged on the use of quartz glass crucibles [Fig. 1(a)], made from modified quick-fit glassware over traditional alumina crucibles. Alumina crucibles are perhaps the most ubiquitously used vessel in the literature of solid-state syntheses to contain volatile reactants like $\mathrm{Bi}_{2} \mathrm{O}_{3}$, but it was found that when attempting to make BFCO, no perovskite phase could be formed, even when burying a pellet inside extra unreacted powders. We believe our quartz vessels have superior performance over alumina due to the higher than ambient internal pressure afforded by the tight quick-fit seal. Sintering in these quartz vessels for short reaction times at an optimal temperature of $860^{\circ} \mathrm{C}$ and then performing a quick quenching to air resulted in the formation of a dominant rhombohedrally distorted perovskite phase that was verified by X-ray Powder Diffraction (XRPD) [Fig. 1(b)].

Such a dominant phase could be indexed to a rhombohedral structure inherited from the $\mathrm{BiFeO}_{3}$ parent phase, in agreement with BFCO, synthesized under high pressure. ${ }^{10,12}$ In addition, no $c$-glide breaking, $\langle 1 / 2,1 / 2,1 / 2\rangle_{\mathrm{P}} *$ pseudo-cubic peaks were visible in the low $2 \theta$, high $d$ region of the XRPD pattern [around $19.4^{\circ}$ for $\mathrm{Cu} \mathrm{K}_{\alpha}$, see Fig. 1(b) (inset)] to indicate that the sample possesses the ordered double-perovskite structure (R3, O-phase). However, due to the extremely similar X-ray form factors of $\mathrm{Fe}$ and $\mathrm{Cr}$, the dominant $\mathrm{Bi}$ form factor, and the large $\mathrm{Fe}$ fluorescence, observation of such reflections is incredibly difficult. For these reasons, conclusions as regards the $\mathrm{Fe} / \mathrm{Cr}$ chemical ordering of $\mathrm{BFCO}$ have not been made based on XRPD data alone.

In Fig. 1(b), we also observe weak impurity signals, with the intensity ratio of the main impurity peak to the main 
BFCO peak being 0.07. It is worth mentioning that by our synthesis approach, several competitive factors would determine the identity of these impurities: the nature of the reducing environment caused by the seal restricting airflow, temperature, and time. Depending on the synthesis conditions, we do observe either oxide/s (e.g., $\mathrm{Bi}_{2} \mathrm{O}_{3}$ or $\mathrm{Bi}_{2} \mathrm{Fe}_{4} \mathrm{O}_{9}$ and $\mathrm{Cr}_{2} \mathrm{O}_{3}$ ) or metal impurities. Under optimal conditions, as we presented in this manuscript, we only see metal $\mathrm{Bi}$ and $\mathrm{Cr}_{2} \mathrm{O}_{3}$ impurities.

\section{B. Search for Fe/Cr chemical ordering}

The theoretically predicted $\left(R 3, a_{\mathrm{R}}=5.47 \AA, \quad \alpha_{\mathrm{R}}\right.$ $=60.09^{\circ}$ ) fully $\mathrm{Fe} / \mathrm{Cr}$ ordered, O-phase has the formula $\mathrm{Bi}_{2} \mathrm{FeCrO}_{6}$ and exhibits regularly alternating $\mathrm{Fe}$ and $\mathrm{Cr}$ along the $[111]_{\mathrm{R}} \equiv[111]_{\mathrm{P}}$ pseudo-cubic parent direction [Fig. 2(a) ]. By contrast, the $R 3 c, \mathrm{Fe} / \mathrm{Cr}$ fully disordered, D-phase has a stoichiometry $\mathrm{Bi}\left(\mathrm{Fe}_{0.5} \mathrm{Cr}_{0.5}\right) \mathrm{O}_{3}$ structure type with the perovskite $B$-site ideally randomly occupied by $\mathrm{Fe}$ and $\mathrm{Cr}$ [Fig. 2(b)]. The $c$-glides in the D-phase structure mean that reflections of the type $[h h l]_{\mathrm{R}} *[l h h]_{\mathrm{R}} *$ and $[h l h]_{\mathrm{R}} *$ (observed at the $\langle 1-10\rangle_{\mathrm{R}} \equiv\langle-110\rangle_{\mathrm{p}}$ zone axis orientations) all have zero intensity unless $l$ is even. As discussed above, it also means that all four $\langle 1 / 2,1 / 2,1 / 2\rangle_{\mathrm{P}} *$ pseudo-cubic, parent reflections (i.e., $\mathbf{a}_{\mathrm{R}} *=[100]_{\mathrm{R}} * \equiv{ }^{l} / 2[-111]_{\mathrm{p}} *, \mathbf{b}_{\mathrm{R}} *=[010]_{\mathrm{R}} * \equiv{ }^{l} / 2$ $[-111]_{\mathrm{p}} *, \quad \mathbf{c}_{\mathrm{R}} *=[001]_{\mathrm{R}} * \equiv l / 2 \quad[11-1]_{\mathrm{p}} *$, and $\mathbf{a}_{\mathrm{R}} *+\mathbf{b}_{\mathrm{R}} *$ $+\mathbf{c}_{\mathrm{R}} *=[111]_{\mathrm{R}} * \equiv 1 / 2[111]_{\mathrm{p}} *$ ) are forbidden by $R 3 c$ space group symmetry. In the $\mathrm{O}$-phase structure, where the $c$-glides are no longer present, these reflections are, of course, no longer forbidden. Detection of these intrinsically weak peaks in XRPD is usually taken as proof of $\mathrm{Fe} / \mathrm{Cr}$ ordering. While our XRPD patterns show an apparent absence of these peaks, their intrinsically weak nature means that they would be difficult to observe anyway. Selected area electron diffraction is much more sensitive to weak features of reciprocal space and was thus performed to search for further evidence of order or disorder.

Given the rhombohedral (but very close to metrically cubic) structures, it would be very difficult to distinguish a $\langle 1-10\rangle_{\mathrm{R}} \equiv\langle-110\rangle_{\mathrm{p}}$ zone axis in the electron diffraction pattern (EPD) from the rhombohedrally distinct $\langle 001\rangle_{\mathrm{R}}$ $\equiv\langle 110\rangle_{\mathrm{p}}$ zone axis for the disordered $R 3$ structure [see, e.g., Fig. 2(c) where the EDP is indexed within experimental error as a $[1-10]_{\mathrm{R}} \equiv[-110]_{\mathrm{p}}$ zone axis (in green) or as a $[001]_{\mathrm{R}}$ $\equiv[110]_{\mathrm{p}}$ zone axis (in red)]. Note that the angle brackets in the previous sentence are used to indicate the set of three, $R 3$ or $R 3 c$ symmetry equivalent, zone axis orientations in each case. In the case of the former indexation, note the arrowed (in green) rows of $[h h l]_{\mathrm{R}} *$ satellite reflections should all be missing if $R 3 c$ were the correct space group symmetry. In the partially, or fully, ordered $R 3$ case, such reflections are in general allowed. Of course, if the red indexing, corresponding to an $[001]_{\mathrm{R}} \equiv\langle 110\rangle_{\mathrm{p}}$ zone axis orientation is correct then there would be no systematic extinction condition of the $\mathrm{F}[h h l]_{\mathrm{R}}{ }^{*}=0$ unless $l$ even observed. We must stress that due to the difficulty in identifying the actual zone axis, and in ensuring that only a single crystal region is illuminated in any particular grain during measurement, this observation does (a) O-Phase

(b) D-Phase
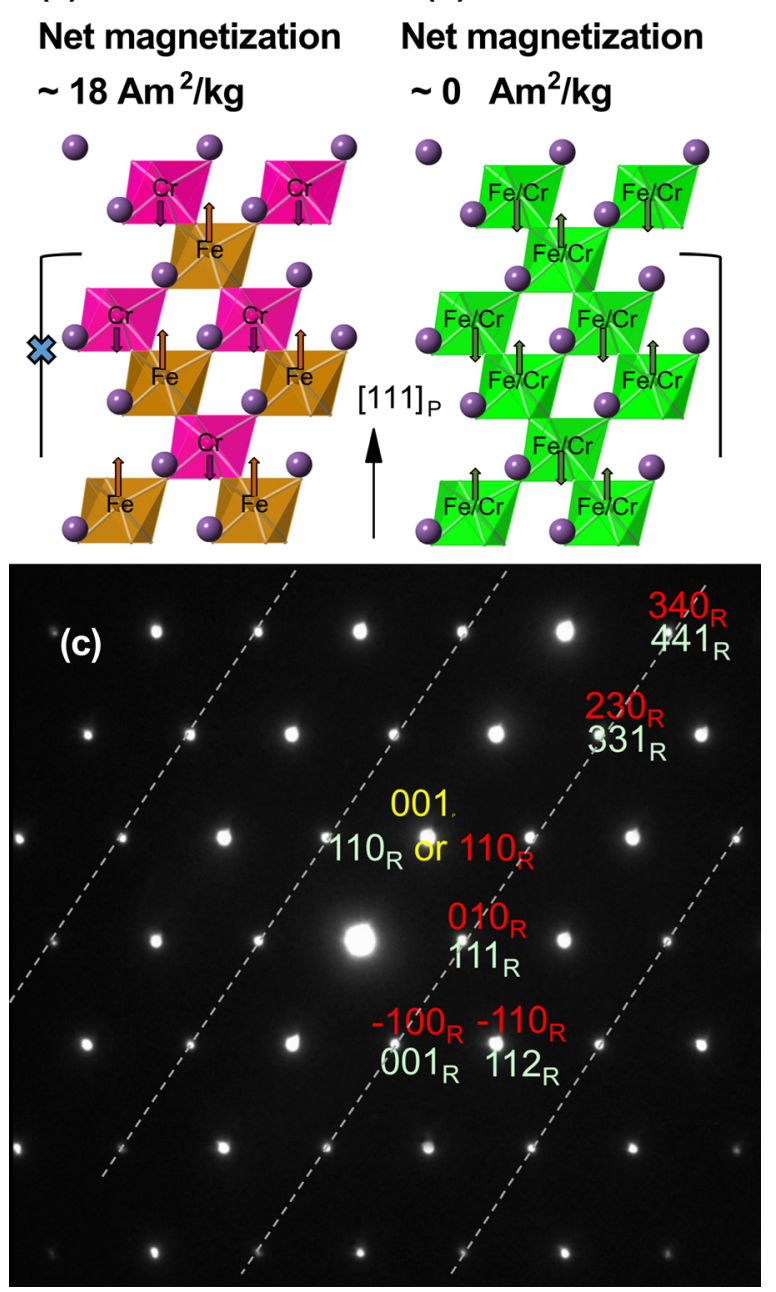

FIG. 2. (a) $R 3$ "O-phase" BFCO structure showing alternate $\mathrm{Fe}$ and $\mathrm{Cr}$ occupation of the octahedral $B$-site along $[111]_{\mathrm{P}}$, with absence of the $c$-glide operation indicated by the cross. Antiferromagnetic coupling of chemically ordered planes leads to a net magnetization of $\sim 18 \mathrm{Am}^{2} / \mathrm{kg}$. (b) Graphical representation of the $R 3 c$ "D-phase" $\mathrm{BFCO}$ structure showing random occupation of the $B$-site and the presence of the $c$-glide. Antiferromagnetic coupling of chemically disordered planes should lead to near zero net magnetization. (c) Electron diffraction pattern (EPD) collected along a typical $\langle 1-10\rangle_{\mathrm{p}}$ zone axis, of which there are six possible such zone axes. Three of these six zone axes will look like what is shown above. The unique three for distinguishing $R 3 c$ from $R 3$ are the $\langle 1-10\rangle_{\mathrm{R}}=\langle-110\rangle_{\mathrm{p}}$ (primitive setting of rhombohedral). The two crystallographically distinct, indexation choices (i) $\langle 1-10\rangle_{\mathrm{R}} \equiv\langle-110\rangle_{\mathrm{p}}$ zone axis edp's (green labelling) and (ii) $\langle 001\rangle_{\mathrm{R}}$ $\equiv\langle 110\rangle_{\mathrm{p}}$ zone axis edp's (red labelling) are given. The fact that the $\langle\mathrm{hhl}\rangle_{\mathrm{R}}{ }^{*}$ rows of reflections (dashed green lines) were always present suggests, but not definitively proves, that the $c$-glide extinction is broken.

not definitively rule out the possibility of the completely disordered $R 3 c$ structure.

Given the specific difficulties in resolving scattering factors and lattice parameters in this material using XRPD and electron diffraction, the diffraction technique with the best chance of detecting definitive evidence for the fully disordered, O-phase is neutron powder diffraction. Neutron diffraction has much greater potential for elemental resolution given that the scattering lengths for $\mathrm{Fe}$ and $\mathrm{Cr}$ are significantly different $(\mathrm{Fe}=9.45 \mathrm{fm}$ and $\mathrm{Cr}=3.64 \mathrm{fm})$ and comparable in magnitude to $\mathrm{Bi}(8.532 \mathrm{fm})^{26}$ allowing the $\langle 100\rangle_{\mathrm{R}} * /[111]_{\mathrm{R}} *$ peak arising from full $B$-site ordering to be easily seen. An added bonus 
of using neutrons is that information on the magnetic structure of the material can also be gained. Room temperature neutron powder diffraction, like XRPD, reveals a rhombohedral phase with lattice parameters $a=b=5.5574(2) \AA$ and $c=13.7444(5) \AA$ (refined in the hexagonal setting for ease). Using the superior elemental scattering factor contrast of this technique, impurities of $\mathrm{Bi}$ metal at $\sim 9 \mathrm{wt} . \%$ and $\mathrm{Cr}_{2} \mathrm{O}_{3}$ at $\sim 5$ wt. $\%$ could be detected. The most interesting feature of the neutron data is an additional signal that is observed in the high $d$-spacing region of the pattern.

Rietveld refinement of a chemically disordered structure in the $R 3 c$ space group yields an excellent fit to the main phase with the exception of a strong reflection at a $d$-spacing of $4.53 \AA$. This peak position is consistent with the previously mentioned $\langle 100\rangle_{\mathrm{R}}{ }^{*} /[111]_{\mathrm{R}}{ }^{*}$ peak. To assess whether this "forbidden" reflection (which was not seen in X-ray diffraction) appears as a result of chemical or magnetic ordering, two further refinements were carried out. The first involved a chemically ordered cell in space group $R 3$ and produced an excellent fit $\left(\mathrm{R}_{\mathrm{wp}}=1.67 \%\right)$ [Fig. 3(a)]. In this model, the occupation of the $\mathrm{Fe}$ and $\mathrm{Cr}$ sites was allowed to vary, though the chemical segregation remained almost complete with the refined occupancy of site one being 0.807(7) $\mathrm{Cr} / 0.193$ (7) $\mathrm{Fe}$ and site two 0.95(1) $\mathrm{Fe} / 0.05$ (1) Cr. It should also be noted that refinement of these occupancies and the identification the $\mathrm{Cr}_{2} \mathrm{O}_{3}$ impurity indicated that this sample

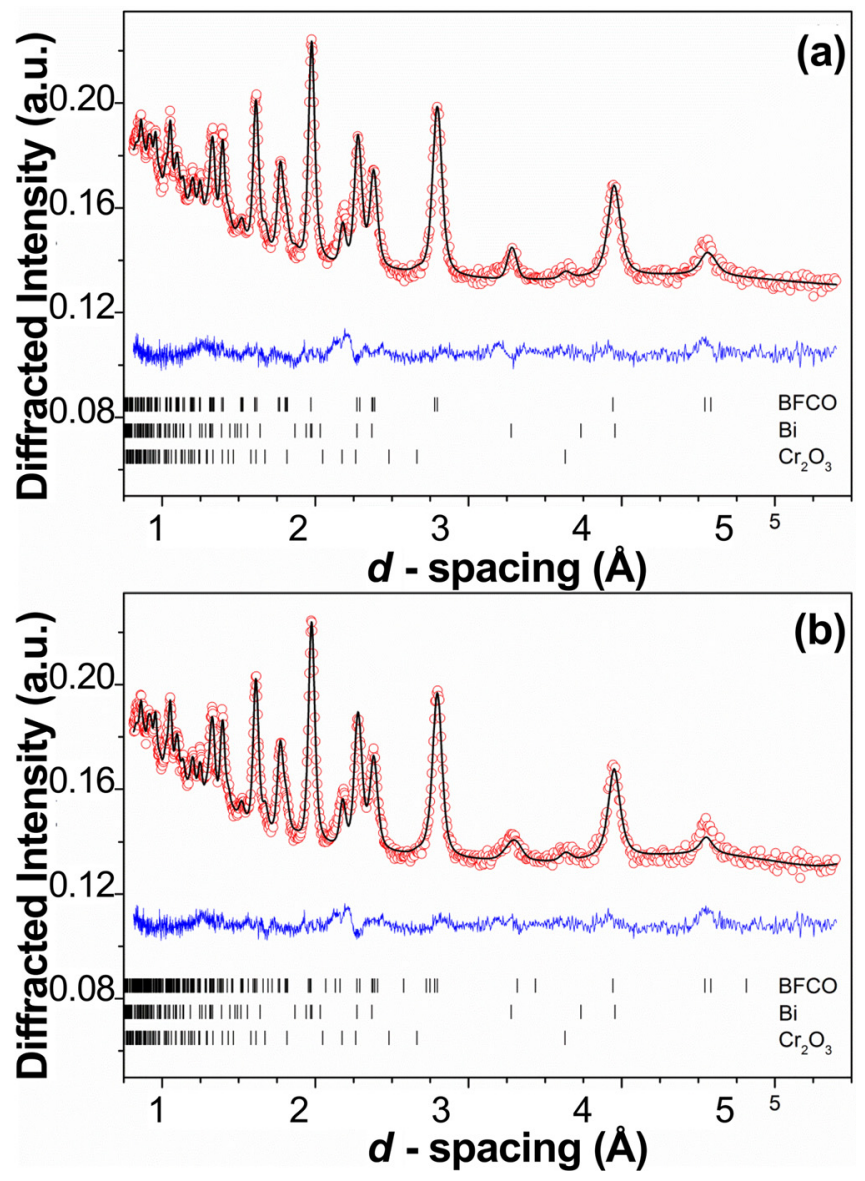

FIG. 3. Room temperature neutron diffraction pattern refined using (a) a chemically ordered $R 3$ space group and (b) a chemically disordered $R 3 c$ space group with magnetic ordering. deviates from the nominal 1:1 $B$-site stoichiometry, and so the chemical formula is estimated to be closer to $\mathrm{BiFe}_{0.57} \mathrm{Cr}_{0.43} \mathrm{O}_{3}$.

The second refinement involved adding magnetic ordering to the chemically disordered $R 3 \mathrm{c}$ model [Fig. 3(b)]. In this model, the $d=4.53 \AA$ peak is caused by antiferromagnetic or ferrimagnetic ordering of the $\mathrm{Fe} / \mathrm{Cr}$ ions described by a $\langle 1 / 21 / 21 / 2\rangle_{P}$ propagation vector instead of chemical ordering. Again, an excellent fit $\left(\mathrm{R}_{\mathrm{wp}}=1.67 \%\right)$ was obtained with a refined magnetic moment of $\sim 0.21 \mu \mathrm{B}$ per $\mathrm{Fe} / \mathrm{Cr}$ atom, representing the moment obtained from the magnetic structure refinement using a G-type antiferromagnetic model. This refined moment value per ion is lower than that would be obtained from a simple average of expected moments for $\mathrm{S}=5 / 2$ and $\mathrm{S}=3 / 2$ ions, pointing towards a degree of frustration in our system. This is not unexpected based on the large range of competing interactions one could have in a chemically random cell (discussed further in Sec. III C).

Based on the room temperature neutron data alone, it is clearly impossible, statistically, to determine which of the fully chemically ordered model with no magnetism [Fig. 3(a)] and the fully chemically disordered plus G-type antiferromagnetism model [Fig. 3(b)] is correct, or indeed whether the true structure contains a degree of both chemical and magnetic ordering. Any attempts to add a magnetic moment into the chemically ordered structure resulted in an overestimation of the $d=4.53 \AA$ peak intensity unless the site occupancies were allowed to refine in order to counteract this. Since the peak of interest is visible only in the poorer resolution lower-angle detector banks of Polaris, it is not possible to meaningfully refine both chemical order and magnetic order parameters simultaneously.

In order to exclude the possibility of partial chemical ordering and be more definitive in the assessment of the structure being D-phase, assessing temperature dependent magnetic and neutron data is necessary. Foreseeably, if the peak is in fact entirely magnetic, when going above $T_{N}$ this signal should vanish in the neutron patterns as the magnetic ordering is lost. The initial simulations ${ }^{8}$ and one experimental bulk synthesis ${ }^{12}$ suggest a magnetic transition temperature below room temperature, but many other reports note magnetization at and above room temperature with predicted transitions at $450-600 \mathrm{~K}^{27,28}$

To this end, a Rietveld refinement was carried out using data measured at $500 \mathrm{~K}$, a temperature which according to the muon data (vide infra) lies above $\mathrm{T}_{\mathrm{N}}$. As can be seen from (Fig. 4), the peak at $4.53 \AA$ has entirely disappeared at this temperature and the data can be fitted with the chemically disordered $R 3 c$ model $\left(\mathrm{R}_{\mathrm{wp}}=1.74 \%\right)$. It is also interesting to note that the peaks from the metallic Bi impurity have also disappeared at this temperature. The melting point of $\mathrm{Bi}$ is around $544 \mathrm{~K}$, and given that the sample had initially been heated to $600 \mathrm{~K}$ it seems entirely plausible that the metal had melted and not yet recrystallised when the $500 \mathrm{~K}$ dataset was collected. The complete disappearance of the $d=4.53 \AA$ peak at $500 \mathrm{~K}$ strongly implies that it is entirely magnetic in origin and that the bulk sample is most likely D-phase BFCO. 


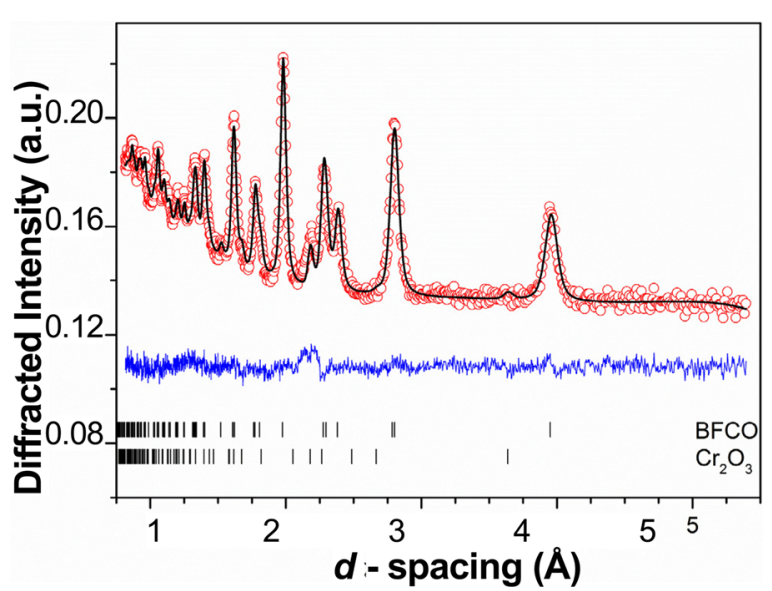

FIG. 4. $500 \mathrm{~K}$ neutron diffraction data and refinement, showing disappearance of $d=4.53 \AA$ peak and Bi phase peaks.

\section{Correlating structure and properties}

One of the critical properties of BFCO predicted in the initial simulation ${ }^{7}$ was the extremely high magnetization. This is closely associated with the assessment of ferrimagnetic ordering arising from the O-phase underlying structure. In order to shed further light on the structural variant of BFCO made in this study and the associated type of magnetism, temperature and field dependent magnetization studies and muon spectroscopy have been performed.

According to the Goodenough-Kanamori-Anderson rules, we would expect antiferromagnetic superexchange between $\mathrm{Fe}^{3+}-\mathrm{O}-\mathrm{Fe}^{3+}$ and $\mathrm{Cr}^{3+}-\mathrm{O}-\mathrm{Cr}^{3+}$ pairs in the disordered $\mathrm{BFCO}$ structure, but ferromagnetic interactions between $\mathrm{Fe}^{3+}-\mathrm{O}-\mathrm{Cr}^{3+}$ pairs. Therefore, in a chemically random cell, the majority of pairings between two ions will be AFM in nature but some will be FM. If we consider the $\sim 156^{\circ} \mathrm{M}-\mathrm{O}-\mathrm{M}$ bond angle we obtain from refinement which would weaken FM interactions of $\mathrm{Fe}-\mathrm{O}-\mathrm{Cr}$ pairs somewhat, and the AFM interactions between $\mathrm{Fe}$ and $\mathrm{Cr} \mathrm{t}_{2 \mathrm{~g}}$ orbitals, ${ }^{29} \mathrm{a}$ frustrated, but overall AFM structure is permitted. Indeed, from refinement of the neutron data, we note magnetic peaks associated with an AFM type magnetic structure but with small refined moments that point towards magnetic frustration. This leads to a very complicated scenario for interpreting temperature and field dependent magnetic behaviour when there are competing local interactions.

Field dependent magnetic hysteresis loops at several temperatures [Fig. 5(a)] show a predominantly linear response with a small inner hysteresis. This magnetization does not saturate even under the maximum applied field and the highest observed magnetization at room temperature is $\sim 0.8 \mathrm{Am}^{2} / \mathrm{kg}(7 \mathrm{~T})$. The linear non-saturating response is typical for an antiferromagnetic material with strong superexchange interactions and the small inner hysteresis is a signature of a weak-ferromagnetic component that is common to many chromites and ferrites including $\alpha-\mathrm{Fe}_{2} \mathrm{O}_{3}{ }^{30}$ and $\mathrm{BiFe}_{1-\mathrm{x}} \mathrm{Mn}_{\mathrm{x}} \mathrm{O}_{3} .{ }^{31,32}$ The weak ferromagnetic signal is commonly attributed to "spin canting" fostered by the Dzyaloshinskii-Moriya ${ }^{33}$ interaction arising from the lack of the inversion centre between each oxygen-metal bond, connected with a distorted bond which is not 180 degrees due to octahedral tilting. ${ }^{34}$ This feature appears to be intrinsic and the signal barely changes with temperature. In fact, the full curves show barely any changes in the temperature range $10-300 \mathrm{~K}$, indicating that the majority phase is strongly ordered at $300 \mathrm{~K}$, well-beneath its Néel temperature $\mathrm{T}_{\mathrm{N}}$, and any paramagnetic impurities (which would produce a $M \alpha$ $1 / T$ dependency) have a negligible effect. The low saturation magnetization observed does not match the expected longrange ferrimagnetic response one would see in the $\mathrm{O}$-phase. In the O-phase, each $\mathrm{Fe}-\mathrm{Cr}$ pair should produce $\sim 2 \mu \mathrm{B}$ moments resulting in large magnetizations near $18 \mathrm{Am}^{2} / \mathrm{kg}$ in the ground state. Instead, the observed magnetization is only $\sim 1.0 \mathrm{Am}^{2} / \mathrm{kg}$ under the highest field tested $(7 \mathrm{~T}, 10 \mathrm{~K})$, or $0.106 \mu \mathrm{B} / \mathrm{Fe}-\mathrm{Cr}$ pair, which is more in line with expections for a more antiferromagnetic system.

The temperature dependent magnetization [Fig. 5(b)] of this material is quite complex and at least partly complicated by the fact that magnetic impurities are present. We can note though that the field-cooled (FC) and the zero-field cooled (ZFC) data diverge below room-temperature which strongly indicates a $\mathrm{T}_{\mathrm{N}}>325 \mathrm{~K}$. The irreversible magnetization that is evident in the difference between FC and the zero-field cooled ZFC spectra of the sample may be explainable by the proposed small levels of spin canting that also give rise to the previously mentioned weak hysteresis in the $\mathrm{M}-\mathrm{H}$ data. Below $20 \mathrm{~K}$, we see a feature that is consistent with the presence of antiferromagnetic interactions [reciprocal of Fig. 5(b) can be extrapolated to the negative temperature axis, not shown]. As noted earlier, in a chemically random cell there are many options for local interactions, and we predict frustrated antiferromagnetism. However, this feature is influenced by the neighbouring broad feature at $\sim 100 \mathrm{~K}$. Other ferrite systems of the type $\mathrm{RFeO}_{3}{ }^{35,36}$ exhibit similar features which are attributed to spin reorientations and $\mathrm{LaCoO}_{3}$ exhibits complex spin transition in a similar region. ${ }^{37}$ However, we are hesitant to ascribe this feature in the low temperature data as such due to the sensitivity of the MPMS technique to impurities. The $>300 \mathrm{~K}$ data should be less influenced by impurities, given the $\mathrm{T}_{\mathrm{N}}$ of $\mathrm{Cr}_{2} \mathrm{O}_{3}$, our main magnetic impurity, is $\sim 308 \mathrm{~K}^{38}$ A separate set of magnetic measurements [Fig. 5(c)] were conducted above room temperature and show a slope change in the susceptibility at approximately $400 \mathrm{~K}$ that appears to indicate a magnetic transition.

In general, the combination of these field and temperature dependent magnetization studies seems to support our manufacturing of a D-phase sample with overall antiferromagnetic type behaviour up to room temperature, in contrast with the calculated ferrimagnetic O-phase. However, magnetization measurements are a bulk technique, and the behaviour is attributable to the entire sample including impurities. Muon Spin Relaxation ( $\mu \mathrm{SR})$ is a local probe, where the muon samples a radius of a few nanometres through dipolar coupling to internal magnetic moments, both nuclear and electronic, associated with the sample. Muons can therefore separate out the behaviour of different phases within the sample.

Throughout the whole temperature range, the muon spectra could be fit using a single exponential relaxation 
(a)

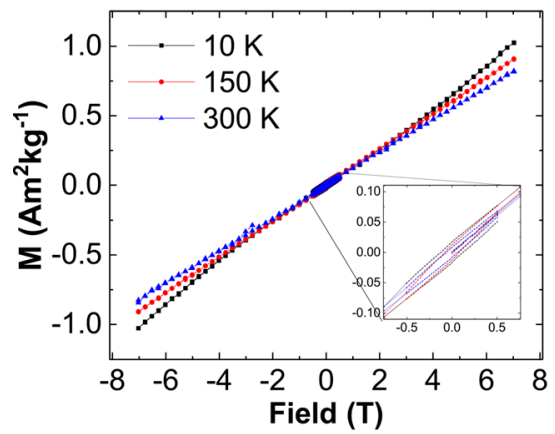

(b)

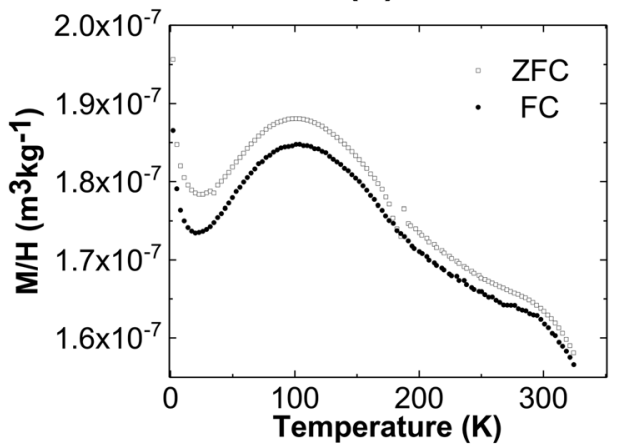

(c)

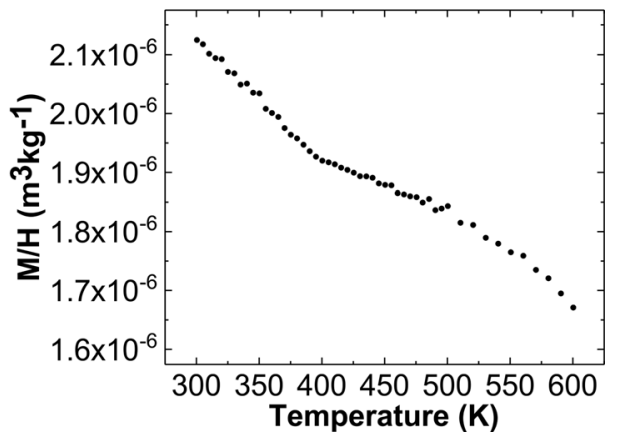

FIG. 5. (a) Field dependent magnetization of BFCO samples at 10 (black), 150 (red), and $300 \mathrm{~K}$ (blue) shows hysteresis at zero field, magnified in an inset and (b) temperature dependent magnetization under a $1 \mathrm{~T}$ applied field, zero field cooled (ZFC, open squares) and field cooled (FC, closed circles). (c) High temperature field cooled magnetization data at $0.0175 \mathrm{~T}$, indicating possible $\mathrm{T}_{\mathrm{N}}$ at $\sim 400 \mathrm{~K}$.

$$
G(t)=A e^{-\lambda t}+A_{0},
$$

where $A$ is the asymmetry associated with the sample, $\lambda$ is the muon relaxation, and $A_{0}$ is the baseline that arises from muons stopping in the sample holder or cryostat, which in this case is $11.7 \%$ of them. Since the sample was small, the fly-past setup was used. The small mass of the sample may also lead to a situation where muons pass through the sample and stop in the backing plate, which can increase the baseline from the nominal value of $4 \%$. However, it cannot be discounted that the high baseline comes from muons stopping in regions of the sample that contain cancelling fields or are non-magnetic and so there would be no dephasing of the muon polarization.

Figure 6 shows the results from the fits to the data from 100 to $460 \mathrm{~K}$. There appears to be a maximum in $\lambda$ at the lowest temperatures, which likely corresponds to a transition in which electronic magnetic fluctuations freeze out. The trend in $\lambda$ with respect to temperature reflects that observed in the magnetization-temperature (M-T) data collected by a magnetic property measurement system (MPMS), though the low temperature peak is shifted to above $200 \mathrm{~K}$. This transition might be a frequency dependent process, and the faster speed of the Muon probe in comparison with MPMS accounts for the shift. These fluctuation features are observed below the minimum in the asymmetry, which could indicate that there is a dynamic component transverse to the $z$-direction. With the increase in temperature, $\lambda$ falls off as the electronic fluctuations become faster. As mentioned, there are no changes in the asymmetry until $300 \mathrm{~K}$, where there is then a dramatic increase and recovery of a missing fraction. A missing fraction occurs when the internal field at the muon site is large enough that it dephases the muon ensemble outside of the measureable time scale. In this case, this would be $10 \mathrm{MHz}$ or $0.08 \mathrm{~T}$. This reduction in the asymmetry along with a peak in $\lambda$ is typical for a magnetically ordered system, where the fluctuations freeze out on the experimental time scale. By $400 \mathrm{~K}$, the relaxing asymmetry has almost flattened out but, temperatures above this could not be reached due to limitations of the cryogenic equipment. Since the relaxing asymmetry is flat below $300 \mathrm{~K}$, this makes the value a good estimate of $T_{\mathrm{C}}$ for fitting. When one attempts to extract the dynamical exponent, $\varpi$ [see Fig. 6(b)], using the equation

$$
\lambda=D\left(\frac{T}{T_{C}}-1\right)^{-\pi}
$$

where $D$ is a prexponent and $\varpi$ is the dynamical exponent, the value equates to $0.31(3)$, which is close to the value of 0.32 , the expected value for a $3 \mathrm{D}$ antiferromagnetic Heisenberg system.

The fact that the muon relaxation at low temperatures is not flat suggests that there is either a component of the magnetization that is slowly fluctuating, or that the field distribution may be fairly broad. The relaxing asymmetry at the lowest temperatures is almost $1 / 3$ of that at high temperatures; this is also expected as the magnetization in a powdered sample will have a $1 / 3+1 / 3+1 / 3$ directional average, and so the data fit with the idea that the sample shows bulk ferromagnetic or antiferromagnetic behaviour, as the (a)

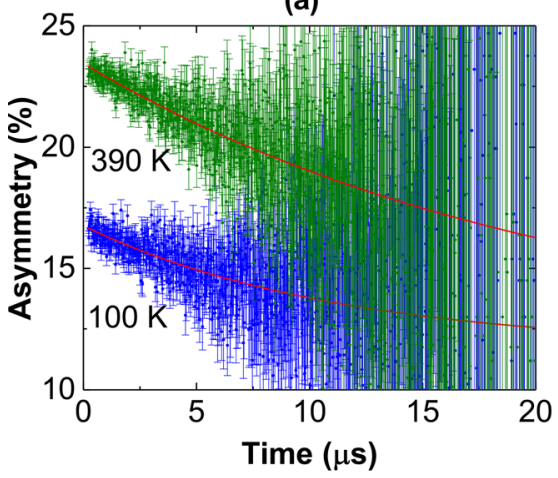

(b)

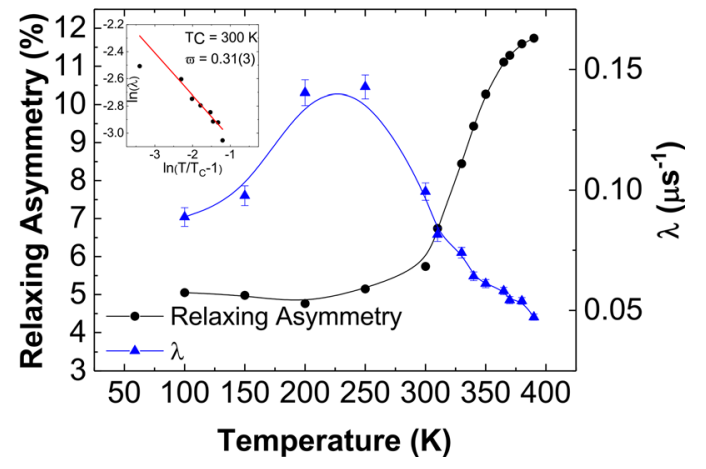

FIG. 6. (a) The raw muon spectra at 100 and $390 \mathrm{~K}$, the solid lines are the fits to the data. (b) The relaxing asymmetry and muon relaxation, $\lambda$, as a function of temperature. Inset: Critical plot where TC was fixed at $300 \mathrm{~K}$ to get the dynamical exponent associated with the transition. 
dynamic exponent suggests. Combining the observations of apparent magnetic transitions in the high temperature muon relaxation, magnetization, and neutron diffraction data, we conclude that BFCO is magnetically ordered at room temperature, in a manner which is compatible with the D-phase BFCO structure.

Note that this bulk BFCO ceramic sample is not very dense, which limits the electric field that can be applied on it. For completeness, the multiferroic nature of the sample has also been assessed with piezoresponse force microscopy (PFM). Distinguished from its morphology [Fig. 7(a)], both the amplitude and phase images suggest the existence of $180^{\circ}$ domains [Figs. 7(b) and 7(c)], which are electrical field-switchable as shown in Figs. 7(d) and 7(e), presenting a typical ferroelectric feature. The simultaneous existence of the antiferromagnetic ordering and the ferroelectric polar response suggests that this BFCO sample is multiferroic.

\section{Rationalizing the result}

From the combined experimental characterizations, we have shown that our simple synthesis method results in a multiferroic phase. This phase however is dissimilar to the theoretical predictions in both structure and properties. In this section, a theoretical study is presented to highlight possible reasons for this result.

Using the results from the room temperature neutron powder pattern refinement, bond valence calculations were performed on the ordered and disordered cells using the SoftBV ${ }^{20,21}$ software. Using the experimental lattice constants and the refined atomic positions, the global instability index (GII) for the disordered cell was calculated to be 0.1346 valence units (v.u.), whereas the ordered cell produces a GII of 0.1445 v.u. The bond valence sum calculations also suggest that each ion is very comfortable with the expected oxidation states $\mathrm{Bi}^{3+}, \mathrm{Fe}^{3+}, \mathrm{Cr}^{3+}$, and $\mathrm{O}^{2-}$. As the GII is correlated with the total energy of the system, this indicates that the disordered structure may have moderately lower energy, however as the difference is relatively small, both structures are viable.

To elucidate the energetic cost of converting the ordered chemical cell to a partially disordered cell, DFT calculations were performed on the ordered and partially ordered cell containing one $B$-site defect (Fig. 8), of the 6 $B$-sites per cell. In both cases, the antiparallel alignment of spins in neighbouring $B$ sites (i.e., G-type order) was found to be stable. Table I records the lattice parameters, energies, and magnetic moments for the fully ordered and partially disordered structures. For the case of BFCO, the DFT results indicate that the energy difference of the ordered cell and the $B$-site defect is $0.09 \mathrm{eV}(\mathrm{GGA}+\mathrm{U})$. The partially disordered cell is found to be energetically favoured, in qualitative agreement with the bond-valence model calculations. However, the overall energy difference between the fully ordered and disordered state is small. This indicates that high-entropy chemically disordered versions of this oxide will generally be favoured during quenching, although it may be possible to achieve meta-stable local chemical order under specific annealing conditions.
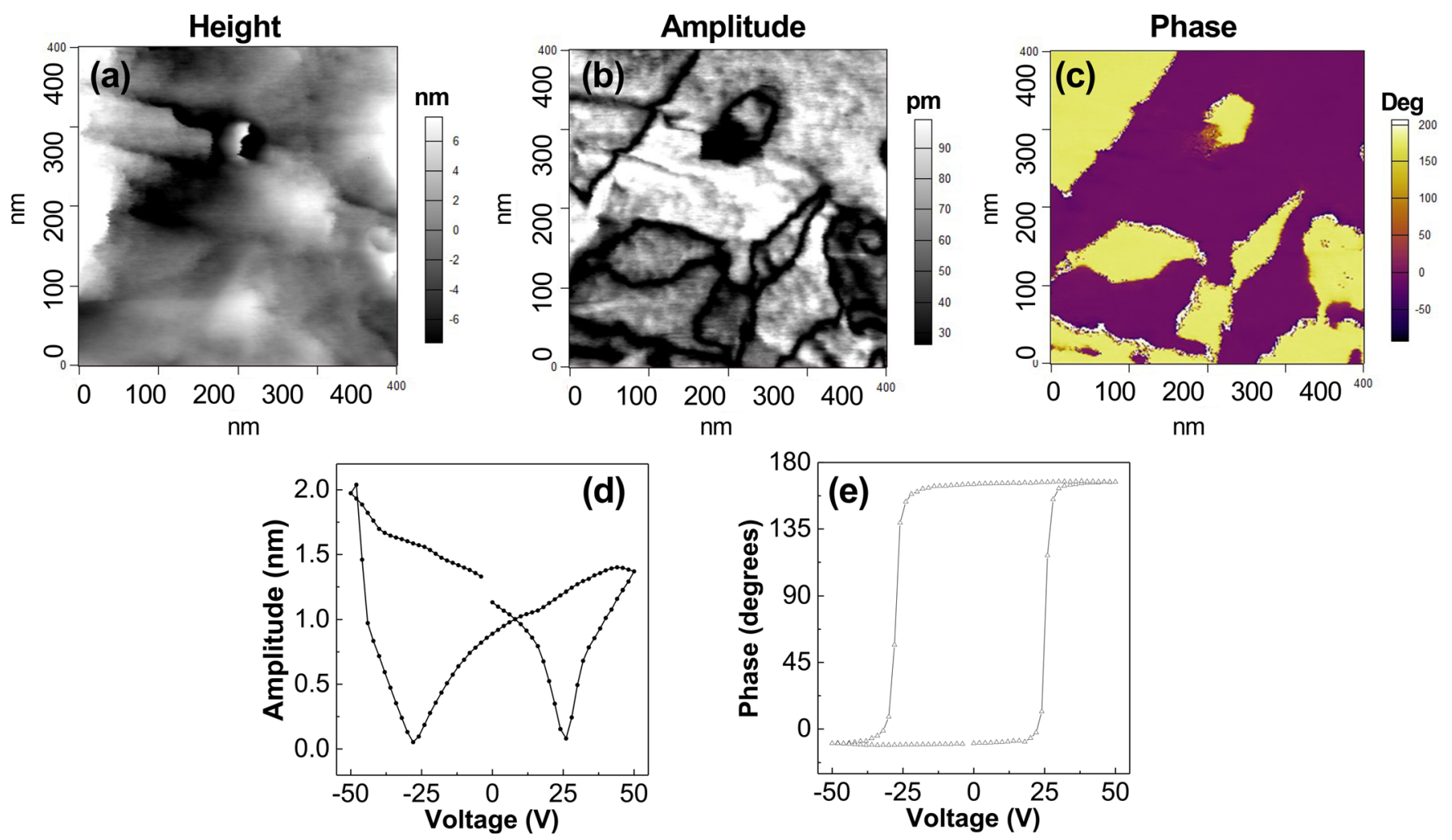

FIG. 7. The surface morphology (a), amplitude (b) and phase (c) images of BFCO bulk ceramic samples characterized by PFM. (d) and (e) are amplitude and phase switchable under an extrinsically applied electric field, in which the domain can be switched $180^{\circ}$, and the strain also varies correspondingly, presenting features typical of the ferroelectric property. 


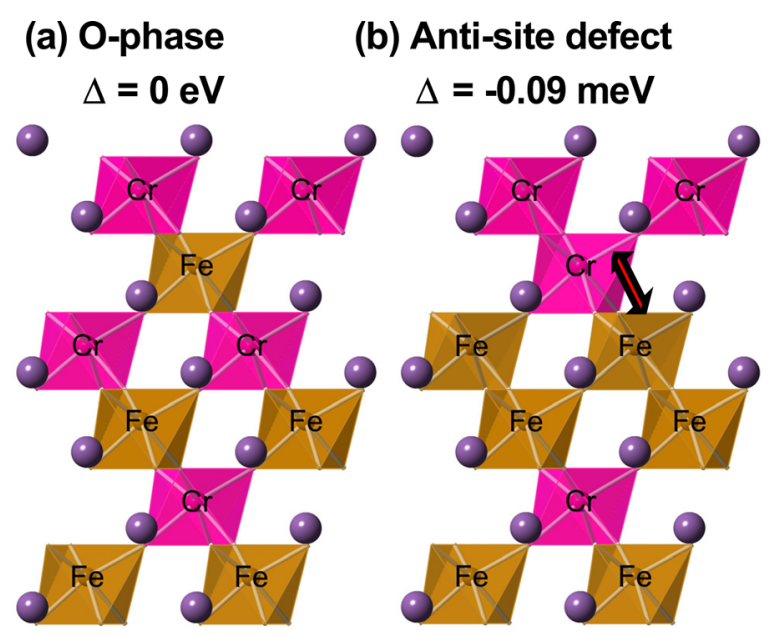

FIG. 8. (a) shows the ordered BFCO structure and (b) a BFCO structure modified with an anti-site defect. GGA + U results give (b) $0.09 \mathrm{meV}$ lower energy.

The lattice parameters calculated in DFT are expanded by $2.5 \%$ compared to the experiment. This is standard agreement for the PBE-GGA method. ${ }^{39}$ On the other hand, the calculated lattice constants differ by up to $20 \%$ compared to the past calculation by Spaldin et al. Further investigation reveals that the details depend on the degree to which the cell shape is preserved during the relaxation, and where the $c: a$ ratio was allowed to vary. Where the $c: a$ ratio was allowed to vary freely, it was found that the chemically ordered BFCO tends to collapse to very small $c$ parameters, matching those in the earlier work. Although the ionic relaxation was performed in the lowest possible symmetry $(P 1)$, the symmetry of the resulting configurations was tested to determine if higher symmetries emerged after relaxation. For both chemically ordered and disordered cells, it was found that the system has three-fold symmetry. The chemically ordered cell is very close to the ideal $R 3$ symmetry within a tolerance of $0.05 \AA$. For the chemically disordered state, the anti-site defect breaks symmetry locally to introduce a local P3 structure. It should be noted that our calculation results may slightly differ from previous reports as a consequence of our differing set-up (e.g., unit cell) and our exchangecorrelation functional choice. This will not affect the overall trend and conclusion we draw however.

TABLE I. Comparison of experimental and theoretical structures and the resulting magnetic moments in BFCO.

\begin{tabular}{lccc}
\hline \hline & & & \\
& Experiment & DFT-Ordered & $\begin{array}{c}\text { DFT - } \\
\text { p-Disordered }\end{array}$ \\
\hline Relative energy $(\mathrm{eV})$ & $\ldots$ & 0.0 & $-0.09 \mathrm{eV}$ \\
Cell volume $\left(\AA^{3}\right)$ & $367.62(3)$ & 377.07 & 376.52 \\
Lattice a $(\AA)$ & $5.5574(2)$ & 5.604 & 5.601 \\
Lattice c $(\AA)$ & $13.7444(5)$ & 13.863 & 13.856 \\
Emergent symmetry & $R 3 c$ & $R 3$ & $P 3$ \\
Magnetic moment Fe $\left(\mu_{\mathrm{B}}\right)$ & $\sim 0.21$ & 3.76 & $3.73-3.76$ \\
Magnetic moment Cr $\left(\mu_{\mathrm{B}}\right)$ & $\sim 0.21$ & 2.22 & $2.23-2.31$ \\
Net magnetic moment $\left(\mu_{\mathrm{B}}\right)$ & $\mathrm{N} / \mathrm{A}$ & 6.00 & 2.00 \\
per unit cell & & & \\
\hline \hline
\end{tabular}

The ab-initio calculations also demonstrate that chemical disorder has a strong effect on the magnetic properties. In the chemically ordered cell with the experimental lattice constants, the magnetic moment on each of the $\mathrm{Fe}$ and $\mathrm{Cr}$ ions was $3.76 \mu_{\mathrm{B}}$ and $-2.22 \mu_{\mathrm{B}}$, respectively, within the atomic spheres. For G-type magnetic ordering, as postulated from the experiment, the total spin moment in the cell was $6.0 \mu_{\mathrm{B}}$ (which included interstitial regions) translated into $2.0 \mu_{\mathrm{B}}$ per $\mathrm{Fe}-\mathrm{Cr}$ pair in agreement with past work. ${ }^{7,8}$ For the partially chemically disordered cell, the magnetic moments of the $\mathrm{Fe}$ and $\mathrm{Cr}$ ions were calculated at $3.73 \mu_{\mathrm{B}}$ and $-2.23 \mu_{\mathrm{B}}$, respectively, but vary within about $0.02 \mu_{\mathrm{B}}$ depending on the local disorder. With G-type magnetic ordering, the resulting net moment in the partially disordered cell becomes only $2.0 \mu_{\mathrm{B}}$, giving an average of $0.67 \mu_{\mathrm{B}}$ per $\mathrm{Fe}-\mathrm{Cr}$ pair. Comparing the chemically ordered and partially chemically disordered cells, a strong reduction in the net moment is observed. This is the result of magnetic frustration between the minor and major spin sublattices caused by switching iron and chromium between their ordered planes on the $B$-sites. This can be intuitively understood because if the G-type ordering is conserved, one $\mathrm{Fe}-\mathrm{Cr}$ pair contributes $2.0 \mu_{\mathrm{B}}$, whereas the neighbouring $\mathrm{Cr}-\mathrm{Fe}$ pair contributes $-2.0 \mu_{\mathrm{B}}$ leading to cancelation. Thus although the partially disordered cell only contains a single $B$-site defect, it already demonstrates a $66 \%$ reduction in the moment. This reflects the low moment we refine from our experimental neutron data and further supports the notion that we have a chemically disordered, frustrated antiferromagnetic material. We do acknowledge that the constraints on the supercell size prevent exploring other large-scale random configurations in DFT, meaning that it is impossible to rigorously construct the fully disordered cell. On the other hand, past Monte Carlo classical spin simulations have explored the effect of large scale random chemical disorder indicating a high population of anti-site defects in a ferrimagnet ultimately prohibiting long-range ferrimagnetism, and giving almost zero global magnetization, except for small local spin-canting effects. $^{40}$

From these results, it seems reasonable that the difficulty in synthesizing O-phase in the bulk is related to the low energy associated with (partially) disordered BFCO structures. Evidently, obtaining chemical ordering in bulk samples of BFCO is experimentally difficult. Ordered (or partially ordered) BFCO formation is reported in thin, ${ }^{41}$ strained, ${ }^{42}$ and artificially layered films ${ }^{43}$ but not in bulk. It is suspected that the strain developed in thin films allows for deviations from the native bulk structure that changes the energy landscape for chemical ordering. Additionally, delivery of reactants to the surface of a $2 \mathrm{D}$ substrate may help both impose artificial chemical ordering and reduce parasitic phase formation in a way bulk 3D solid state diffusion and quenching cannot. The formation of parasitic phases ultimately affects the stoichiometry and growth of the main phase. To reduce these in the bulk synthesis, we presently invoke quenching which, as mentioned earlier, is likely to cause the formation of the more entropic disordered BFCO phase. Different sintering conditions may lead to the development of order, but to do so, the impurity levels need to be 
addressed lest they increase in fraction. Work is ongoing to address this challenge.

\section{CONCLUSIONS}

A rhombohedral multiferroic phase can be produced from $\mathrm{Bi}_{2} \mathrm{O}_{3}, \mathrm{Fe}_{2} \mathrm{O}_{3}$, and $\mathrm{Cr}_{2} \mathrm{O}_{3}$ at the gram-scale via a nearambient pressure solid-state reaction if a quartz crucible is employed. The material is proven to be multiferroic at room temperature through magnetic and electrical measurements. Temperature dependent magnetometry and muon spin relaxation point to the main BFCO phase being magnetically ordered up to $\sim 400 \mathrm{~K}$. Investigation with neutron powder diffraction at room temperature and $500 \mathrm{~K}$ and subsequent refinement support disordered chemical occupation of the $B$ site with $\mathrm{Fe}$ and $\mathrm{Cr}$ resulting in antiferromagnetic ordering. This is dissimilar to the initial theoretical predictions made in the literature. Our assignment of a disordered structure is rationalized based on ab initio calculations that place this structure as lower in energy.

\section{ACKNOWLEDGMENTS}

B.R.M., J.L., A.B., D.L.C., T.L., R.L.W., N.N., and Y.L. acknowledge the support of the Australian Research Council (ARC) in the form of Discovery Projects (No. DP160104780). A.B., D.L.C., N.N., D.Y. and authors thank the Australian Nuclear Science and Technology Organisation (ANSTO) for facilities and financial support and also thank the Echidna instrument scientists for their expertise. All authors acknowledge the Centre for Advanced Microscopy, Australian National University, for use of their facilities. B.R.M. is financially supported by the Australian Government Research Training Program (RTP) and the ANU Research School of Chemistry Scholarships. We are grateful to the Science and Technology Facilities Council and RIKEN for providing beamtime at RIKEN-RAL. We would also like to acknowledge the ISIS Materials Characterisation Laboratory for access to conduct magnetic measurements as well as Dr. Ian Terry (Durham University) for providing magnetization measurements using the QD oven option.

${ }^{1}$ M. Gajek, M. Bibes, S. Fusil, K. Bouzehouane, J. Fontcuberta, A. Barthélémy, and A. Fert, Nat. Mater. 6, 296 (2007).

${ }^{2}$ G. Catalan and J. F. Scott, Adv. Mater. 21, 2463 (2009).

${ }^{3}$ T. Rojac, A. Bencan, B. Malic, G. Tutuncu, J. L. Jones, J. E. Daniels, and D. Damjanovic, J. Am. Ceram. Soc. 97, 1993 (2014).

${ }^{4}$ S. M. Selbach, M.-A. Einarsrud, and T. Grande, Chem. Mater. 21, 169 (2009).

${ }^{5}$ M. Valant, A.-K. Axelsson, and N. Alford, Chem. Mater. 19, 5431 (2007).

${ }^{6}$ M. Bernardo, T. Jardiel, M. Peiteado, A. Caballero, and M. Villegas, J. Eur. Ceram. Soc. 31, 3047 (2011).

${ }^{7}$ P. Baettig and N. A. Spaldin, Appl. Phys. Lett. 86, 012505 (2005).

${ }^{8}$ P. Baettig, C. Ederer, and N. A. Spaldin, Phys. Rev. B: Condens. Matter Mater. Phys. 72, 214105 (2005).
${ }^{9}$ R. Nechache, C. Nauenheim, U. Lanke, A. Pignolet, F. Rosei, and A. Ruediger, J. Phys.: Condens. Matter 24, 142202 (2012).

${ }^{10}$ J. L. Zhu, H. X. Yang, S. M. Feng, L. J. Wang, Q. Q. Liu, C. Q. Jin, X. H. Wang, L. T. Li, and J. Yu, Int. J. Mod. Phys. B 27, 1362023 (2013).

${ }^{11}$ V. Shabadi, M. Major, P. Komissinskiy, M. Vafaee, A. Radetinac, M. B. Yazdi, W. Donner, and L. Alff, J. Appl. Phys. 116, 114901 (2014).

${ }^{12}$ M. R. Suchomel, C. I. Thomas, M. Allix, M. J. Rosseinsky, A. M. Fogg, and M. F. Thomas, Appl. Phys. Lett. 90, 209901 (2007).

${ }^{13}$ R. Nechache, C. Harnagea, S. Licoccia, E. Traversa, A. Ruediger, A. Pignolet, and F. Rosei, Appl. Phys. Lett. 98, 202902 (2011).

${ }^{14}$ Y. Shimakawa, M. Azuma, and N. Ichikawa, Materials 4, 153 (2011).

${ }^{15}$ F. Chang, N. Zhang, F. Yang, S. Wang, and G. Song, J. Phys. D: Appl. Phys. 40, 7799 (2007).

${ }^{16}$ I. V. Lisnevskaya, T. G. Lupeiko, and E. A. Bikyashev, Russ. J. Inorg. Chem. 60, 140 (2015).

${ }^{17}$ O. Arnold, J.-C. Bilheux, J. Borreguero, A. Buts, S. I. Campbell, L. Chapon, M. Doucet, N. Draper, R. F. Leal, and M. Gigg, Nucl. Instrum. Methods Phys. Res., Sect. A 764, 156 (2014).

${ }^{18}$ A. C. Larson and R. B. Von Dreele, "General structure analysis system (GSAS)," Los Alamos National Laboratory Report LAUR 86-748, 2000.

${ }^{19}$ B. H. Toby, J. Appl. Crystallogr. 34, 210 (2001).

${ }^{20}$ S. Adams, Acta Crystallogr., Sect. B 57, 278 (2001).

${ }^{21} \mathrm{~S}$. Adams, see http://www.softbv.net/ for general information on the soft BV method and the on-line BV calculator tool (2017).

${ }^{22}$ P. Blaha, K. Schwarz, G. Madsen, D. Kvasnicka, and J. Luitz, "An augmented plane wave+ local orbitals program for calculating crystal properties," (Techn. Universität Wien, Austria, 2001).

${ }^{23}$ J. P. Perdew, K. Burke, and M. Ernzerhof, Phys. Rev. Lett. 77, 3865 (1996).

${ }^{24}$ V. I. Anisimov, I. Solovyev, M. Korotin, M. Czyżyk, and G. Sawatzky, Phys. Rev. B 48, 16929 (1993).

${ }^{25}$ J. Silva, A. Reyes, H. Esparza, H. Camacho, and L. Fuentes, Integr. Ferroelectr. 126, 47 (2011).

${ }^{26}$ V. F. Sears, Neutron News 3, 26 (1992).

${ }^{27}$ S. Kamba, D. Nuzhnyy, R. Nechache, K. Zaveta, D. Niznansky, E. Santava, C. Harnagea, and A. Pignolet, Phys. Rev. B: Condens. Matter Mater. Phys. 77, 104111 (2008).

${ }^{28}$ Z.-W. Song and B.-G. Liu, Chin. Phys. B 22, 047506 (2013).

${ }^{29}$ K. Miura and K. Terakura, Phys. Rev. B 63, 104402 (2001).

${ }^{30}$ F. J. Morin, Phys. Rev. 78, 819 (1950).

${ }^{31}$ D. L. Cortie, T. Buck, M. Dehn, V. Karner, R. Kiefl, C. Levy, R. McFadden, G. Morris, I. McKenzie, and M. Pearson, Phys. Rev. Lett. 116, 106103 (2016).

${ }^{32}$ D. L. Cortie, A. P. J. Stampfl, F. Klose, Y. Du, X. L. Wang, H. Y. Zhao, H. Kimura, and Z. X. Cheng, Appl. Phys. Lett. 101, 172404 (2012).

${ }^{33}$ T. Moriya, Phys. Rev. 120, 91 (1960).

${ }^{34}$ C. Ederer and N. A. Spaldin, Phys. Rev. B: Condens. Matter Mater. Phys. 71, 060401 (2005).

${ }^{35}$ S. S. K. Reddy, N. Raju, C. G. Reddy, P. Y. Reddy, K. R. Reddy, and V. R. Reddy, J. Magn. Magn. Mater. 396, 214 (2015).

${ }^{36}$ Y. Fang, Y. Yang, X. Liu, J. Kang, L. Hao, X. Chen, L. Xie, G. Sun, V. Chandragiri, and C.-W. Wang, Sci. Rep. 6, 33448 (2016).

${ }^{37}$ J. Androulakis, N. Katsarakis, and J. Giapintzakis, Phys. Rev. B 64, 174401 (2001).

${ }^{38}$ T. G. Worlton, R. M. Brugger, and R. B. Bennion, J. Phys. Chem. Solids 29, 435 (1968).

${ }^{39}$ P. Haas, F. Tran, and P. Blaha, Phys. Rev. B 79, 085104 (2009).

${ }^{40}$ J. Bertinshaw, D. Cortie, Z. Cheng, M. Avdeev, A. Studer, F. Klose, C. Ulrich, and X. Wang, Phys. Rev. B 89, 144422 (2014).

${ }^{41}$ R. Nechache, C. Harnagea, and A. Pignolet, J. Phys.: Condens. Matter 24, 096001 (2012).

${ }^{42}$ P. C. Rout, A. Putatunda, and V. Srinivasan, Phys. Rev. B 93, 104415 (2016).

${ }^{43}$ N. Ichikawa, M. Arai, Y. Imai, K. Hagiwara, H. Sakama, M. Azuma, Y. Shimakawa, M. Takano, Y. Kotaka, and M. Yonetani, Appl. Phys. Express 1, 101302 (2008). 